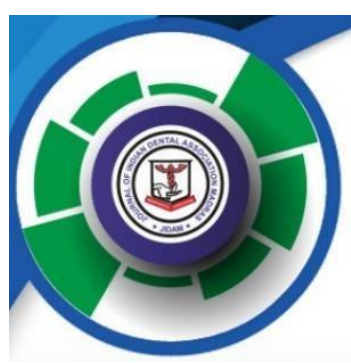

REVIEW ARTICLE

\title{
SHORT IMPLANTS- AN EVOLVING TREND- A REVIEW
}

\author{
Dr. Swetha. T, Dr. Godwin Alex Kiruba*, Dr. Manikandhan Ramanathan**
}

Junior Resident, Department of Prosthodontics, Crown, Bridge and Implantology, Government Dental College and Hospital, Chennai, Tamilnadu, India

*Meenakshi Cleft and Craniofacial Centre, Meenakshi Academy of Higher Education and Research, Chennai, Tamilnadu, India

**Department of Oral and Maxillofacial Surgery, Faculty of Dentistry, Meenakshi Academy of Higher Education and Research, Chennai, Tamilnadu, India

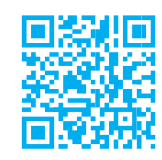

DOI: 10.37841/jidam_2021_V8_I2_05

Address for Correspondence

Dr. Swetha. T, BDS.,

Junior Resident, Department of Prosthodontics, Crown, Bridge and Implantology, Government Dental College and Hospital, Chennai, Tamilnadu, India

Email id: swethat2k14@gmail.com

Received: 23.05.2021 First Published: 07.06.2021

Accepted: 03.06.2021

Published: 27.06.2021

\begin{abstract}
Short implants have been proposed as one of the positive treatment options for compromised edentulous regions. Decreased bone height, poor bone quality often limit the use of conventional implants and usually necessitate complex surgical procedures like sinus lift or bone augmentations which are associated with increased morbidity, treatment time and cost. Though the crown root ratio of short implants is controversial, they have demonstrated a high success rate with innovations that have bolstered its effectiveness through the modifications in micro and macro surface topography, surgical bone drilling protocols and implant diameter. Photo functionalization of implants and customized implants using 3D printing are some of the promising developments in short implants.
\end{abstract}

KEYWORDS: Short Implants, Implant surface topography, Bone grafting, Photo functionalization, Customized Implants 


\section{INTRODUCTION}

Dr. Branemark's discovery of osseointegration in 1952 and the placement of the first Branemark implants in humans in 1965 were the initial landmark events in the field of dental implantology ${ }^{1}$. Since then, this discipline has made great strides in fulfilling the patient's esthetic and functional needs.

\section{WHAT ARE SHORT IMPLANTS?}

Dental implants have been classified by various authors; yet there is no universally accepted consensus as to what constitutes a short or a long dental implant. Many authors have categorically referred to implants with length less than $11 \mathrm{~mm}, 10 \mathrm{~mm}, 8 \mathrm{~mm}$ and $7 \mathrm{~mm}$ as short implants. ${ }^{2,3,4,5}$ There are many trials and systematic reviews in the recent years, which consider implants with length equal to or less than $8 \mathrm{~mm}$ as short implants and the authors have followed the same.

\section{WHY SHORT IMPLANTS WEREN'T PREFERRED?}

Historically, long implants were only preferred on every edentulous areas as the initial trials suggested that the failure rates of short implants were more than long implants. ${ }^{6}$ Most of the earliest documented failures of short implants were seen in areas of poor bone quality with less density, specifically in the posterior maxilla. These trials, though they used turned/machined surface implants, they also had followed the standard drilling protocols of the era. There was no widely accepted protocol to differentiate site preparation for soft versus normal bone.

\section{ADJUNCTS TO SHORT IMPLANTS}

Later, the clinicians began to encounter specific scenarios in which the anatomical constraints prevented the placement of long implants. Pneumatized maxillary sinus with resorbed posterior alveolar ridges, position of the nasal floor, nasopalatine canal, inferior alveolar nerve and mental foramen are some of the potential limitations for placement of long implants. Many advanced surgical techniques were incorporated into clinical practice to counter these limitations, which include maxillary sinus grafting, guided bone regeneration, usage of block bone grafts, distraction osteogenesis and inferior alveolar nerve transposition.

Over the years, the demerits and complications associated with these convoluted and invasive grafting procedures became apparent. A few of these procedures are time consuming, expensive, extremely technique sensitive, necessitate advanced skills and training, with increased surgical morbidity. Jepsen et $\mathrm{al}^{7}$ and Louis et $\mathrm{al}^{8}$ elaborated on the usage and complications of bone grafting materials and techniques with regards to the maxillae. Mazzonetto and colleagues reviewed the complications associated with alveolar distraction osteogenesis ${ }^{9}$.

\section{EVOLUTION OF SHORT IMPLANTS}

There has been a tremendous transformation in implant configuration and biomechanics in the recent years. Advances in the design of dental implants both externally, and internally, the implant to abutment connection have been dramatically transformed. A multitude of improvements in the external implant design including thread design, macro surface, and micro surface topography and in the internal design including implant to abutment connections have enhanced the implant survivability. The development and adoption of modified surgical protocols (soft bone drilling protocols), permit improved primary mechanical stability and have been advocated by several researchers across the globe. ${ }^{9}$ Finite element analyses (FEA) have revealed that increasing the implant diameter is more efficient than increasing implant length for stress reduction at the bone to implant interface. Wider diameter short implants have increased functional surface area (FSA) and improved primary stability. It facilitates the creation of a better emergence profile, specifically in the posterior segment and also increases fracture resistance. ${ }^{10}$ A diameter increase by $1 \mathrm{~mm}$ is supposed to increase the surface area by $30-200 \%$ depending upon the implant design. This better understanding of implant to bone biomechanics has contributed to the increased usage of short implants.

\section{ARE SHORT IMPLANTS BETTER?}

Multiple reviews and trials have been published comparing short implants with long implants in augmented, grafted or modified sites. Thoma et $\mathrm{al}^{11}$ elicited that the short implants in the posterior maxilla had a shorter operative time and were extremely cost-effective. Hazdik and colleagues $^{12}$ published that short implants decreased the need for adjunctive surgical procedures thereby reducing the patient's post-operative pain and morbidity. A few more reviews found no significant difference between the two techniques with regards to implant survival, marginal bone loss and patient satisfaction.

Pieri and colleagues ${ }^{13}$ observed no statistically significant differences in the number of implant or prosthetic failures, prosthetic or biological complications 
Swetha et al: Short Implants- An evolving trend

between long implants placed in augmented sites and short implants in non-augmented sites. The number of surgical complications was greater in the long implants category with the short implants recording a statistically significant lesser marginal bone loss. Annibali et $\mathrm{al}^{14}$ reviewed and elaborated on the utility of short implant-supported prostheses in atrophic jaw and found them to be a valid treatment option. They reported high survival rates and low incidence of biological and biomechanical complications. Surgical technique, implant location, type of edentulism and prosthetic restoration were not observed to affect shortimplant survival.

\section{RECENT ADVANCES}

\section{PHOTO FUNCTIONALIZATION OF IMPLANTS}

Implants that were treated with ultraviolet (UV) light have been found to increase the bone implant contact from $55 \%$ to $98 \%$, thereby increasing the strength of osseointegration. This can be attributed to the generation of superhydrophilicity, improvement in the electrostatic status of titanium surfaces with a significant decrease in surface hydrocarbons. ${ }^{10}$

\section{CUSTOMIZED IMPLANTS USING 3D PRINTING}

Intra-oral scanning with $\mathrm{CAD} / \mathrm{CAM}$ designs coupled with three-dimensional printing had already been used for making surgical guides and customized implant abutments. ${ }^{15}$ We can fabricate customized implants with the analog mimicking the root of the missing tooth. Preoperative CBCT can aid in designing the implant based on the root morphology of the tooth to be extracted, or using the contralateral tooth root morphology in case of a missing tooth. They can offer excellent advantages over the traditional implant designs.

\section{SURFACE TOPOGRAPHY}

Alteration of the implant surface can influence the success of osseointegration. This can be achieved either by subtractive processes like etching, blasting and oxidation, or additive procedures like ion deposition, titanium plasma spraying, calcium phosphate and hydroxyapatite coating. ${ }^{10}$ Implant surfaces treated with silver nanoparticles-based composites have been observed to decrease the biofilm adhesion and lactate production by microorganisms. Implants treated with Silver Plasma have demonstrated antibacterial effects and provide better osteointegration results. ${ }^{16}$

\section{CONCLUSION}

Despite the rarity of HPC, it can be considered as one of the differential diagnosis of tumors of the head and neck region. Though surgical excision is the most accepted management of HPC. Early diagnosis reduces the postsurgical morbidity. Since, local recurrences are very common and late distant metastasis has reported, long-term follow-up, is mandatory both clinically and radiologically.

\section{FINANCIAL SUPPORT AND SPONSORSHIP}

\section{CONFLICT OF INTEREST:}

There are no conflicts of interest.

\section{REFERENCES:}

1. Schwartz SR. Short implant are they a viable option in implant dentistry? Dent Clin North Am 2015; 59:317-28.

2. Neves FD, Fones D, Bernardes SR, et al. Short implants - an analysis of longitudinal studies. Int J Oral Maxillofac Implants 2006; 21:86-93.

3. Morand $\mathrm{M}$, Irinakis $\mathrm{T}$. The challenge of implant therapy in the posterior maxilla: providing a rationale for the use of short implants. J Oral Implantol 2007; 33(5): 257-66.

4. Renouard F, Nisand D. Impact of implant length and diameter on survival rates. Clin Oral Implants Res 2006; 17(Suppl 2):35-51.

5. Friberg B, Jemt T, Lekholm U. Early failures in 4,641 consecutively placed Branemark dental implants: a study from stage 1 surgery to the connection of completed prostheses. Int J Oral Maxillofac Implants 1991; 6:142-6.

6. Schwartz SR. Short Implants: An Answer to a Challenging Dilemma? Dent Clin North Am 2020; 64(2):279-90.

7. Jepsen S, Schwarz F, Cordaro L, et al. Regeneration of alveolar ridge defects. Consensus report of group 4 of the 15th European Workshop on Periodontology on Bone Regeneration. J Clin Periodontol 2019; 46(Suppl. 21):277-86.

8. Louis PJ, Sittitavornwong S. Managing bone grafts for the mandible. Oral Maxillofacial Surg Clin N Am 2019; 31:317-30.

9. Nisand D, Renouard F. Short implant in limited bone volume. Periodontol 2000 2014; 66:72-96.

10. Shetty S, Puthukkat N, Bhat S, Shenoy K. Short implants: a new dimension in rehabilitation of atrophic maxilla and mandible. J Interdis Dent 2014; 
Swetha et al: Short Implants- An evolving trend

4(2).

11. Thoma DS, Haas R, Tutak M, et al. Randomized controlled multicentre study comparing short dental implants $(6 \mathrm{~mm})$ versus longer dental implants (11$15 \mathrm{~mm}$ ) in combination with sinus floor elevation procedures. Part 1: demographics and patientreported outcomes at 1 year of loading. J Clin Periodontol 2015; 42:72-80.

12. Hadzik J, Krawiec M, Kubasiewicz-Ross P, et al. Short implants and conventional implants in the residual maxillary alveolar ridge: a 36month followup observation. Med Sci Monit 2018; 24:5645-52.

13. Pieri F, Forlivesi C, Caselli E, et al. Short implants (6 $\mathrm{mm})$ vs. vertical bone augmentation and standard- length implants $(>9 \mathrm{~mm})$ in atrophic posterior mandibles: a 5-year retrospective study. Int Maxillofac Surg 2017; 46(12):1607-14.

14. Annibali SC, Cristalli MP, Dell'Aquila D, Bignozzi I, La Monaca G, Pilloni A. Short dental implants: a systematic review. J Dent Res 2012; 91(1):25-32.

15. Hong, D.G.K., Oh, Jh. Recent advances in dental implants. Maxillofac Plast Reconstr Surg 2017; 39(1):33.

16. Fernandez CC, Sokolonski AR, Fonseca MS, Stanisic D, Araújo DB, Azevedo V, Portela RD, Tasic L. Applications of Silver Nanoparticles in Dentistry: Advances and Technological Innovation. Int J Mol Sci 2021; 22(5):2485 